\title{
AN ENHANCED PACKET SCHEDULING ALGORITHM FOR THE DOWNLINK COGNITIVE LONG TERM EVOLUTION-ADVANCED
}

\author{
Huda Adibah Mohd Ramli, Mohamad Ismat Hafizi Mansor \\ Department of Electrical and Computer Engineering, \\ International Islamic University Malaysia, \\ P.O. Box 10, 50728 Kuala Lumpur, Malaysia. \\ "Corresponding author: hadibahmr@iium.edu.my
}

(Received: 18 $8^{\text {th }}$ June 2019; Accepted: $30^{\text {th }}$ July 2019; Published on-line: $20^{\text {th }}$ January 2020)

\begin{abstract}
A packet scheduling algorithm known as Enhanced Maximum-Largest Weighted Delay First (EM-LWDF) is proposed in this paper. It aims to maximize the number of Real Time (RT) and Non Real Time (NRT) users that meet their desired Quality of Service (QoS) in the cognitive Long Term Evolution-Advanced (LTE-A). This cognitive LTE-A allows its Component Carriers (CCs) to be combined with licensed CCs from another system. This enables the LTE-A to further expand the available bandwidth for packet transmission. The EM-LWDF algorithm decides the priority of each user on the basis of the desired QoS of each packet, the urgency of each packet, channel quality and the average throughput. The potential of this algorithm is validated via computer simulation where it is able to simultaneously maximize more users that meet their desired QoS in realistic RT and NRT multimedia of the downlink cognitive LTE-A.
\end{abstract}

\begin{abstract}
ABSTRAK: Algoritma penjadualan paket yang dikenali sebagai Enhanced MaximumLargest Weighted Delay First (EM-LWDF) dicadangkan dalam kertas ini. Ia bertujuan untuk memaksimumkan bilangan pengguna Real Time (RT) dan Non Real Time (NRT) yang memenuhi Kualiti Perkhidmatan yang dikehendaki (QoS) dalam system kognitif Long Term Evolution-Advanced (LTE-A). Kognitif LTE-A ini membolehkan Carrier Component (CC) untuk digabungkan dengan $\mathrm{CC}$ berlesen dari sistem lain. Ini membolehkan LTE-A untuk memperluaskan jalur lebar yang tersedia bagi penghantaran paket. Algoritma EMLWDF menentukan keutamaan setiap pengguna berdasarkan QoS yang dikehendaki dari setiap paket, kecemasan setiap paket, kualiti saluran dan purata penghantaran. Potensi algoritma ini disahkan melalui simulasi komputer di mana ia dapat memaksimumkan lebih banyak pengguna yang memenuhi QoS yang dikehendaki mereka dalam realistik RT dan NRT multimedia dari downlink sistem kognitif LTE-A.
\end{abstract}

KEYWORDS: maximum-largest weighted delay first (M-LWDF); packet scheduling; long term evolution-advanced; quality of service; real time and non-real time

\section{INTRODUCTION}

The introduction and evolution of various Real Time (RT) and Non-Real Time (NRT) multimedia content have drastically increased the number of wireless subscriptions. It should be noted that RT content are sensitive to delay whereas NRT is a loss-sensitive multimedia content. To address this scenario, Long Term Evolution-Advanced (LTE-A) that promises a significant increase in the data speed whilst maximizing the number of users that meet their desired Quality of Service (QoS) was standardized. Such increase in the data speed is 
achieved via Carrier Aggregation (CA) method. This method combines a number of Component Carriers (CCs) of the same or different frequency bands.

Given the current escalating demand on the multimedia contents, the limited CCs of the LTE-A will soon be saturated. To offload some portion of the CCs usage, integrating the CCs of the LTE-A with other licensed CCs via Cognitive Radio (CR) seems a feasible solution. It should be noted that the Federal Communication Commission reported in its study that some portions of licensed $\mathrm{CCs}$ are heavily utilized while other portions of the CCs licensed to Digital Video Broadcast (DVB) and TV systems are lightly used [1]. This allows more available CCs to be exploited for use by the LTE-A users. CR is a technology that allows unlicensed users (for example, LTE-A users) to opportunistically utilize the vacant portions of the licensed CCs and quickly release these portions when licensed users (such as DVB users) become active.

Transmission of multimedia content takes place via packet-switched technology. Therefore, packet scheduling is of significant importance in the LTE-A. A large number of packet scheduling algorithms have been developed. For example, the most popular conventional Maximum Largest Weighted Delay First (M-LWDF) [2] algorithm was developed to meet the desired QoS of the RT multimedia content in the single-user legacy wireless systems that have only one CC. The M-LWDF chooses a downlink user to receive its packets based on Eq. (1).

$$
\begin{gathered}
\mu_{k}(i)=a_{k} * R T_{k}(i) * \frac{c q_{k}(i)}{A T_{k}(i)} \\
a_{k}=\frac{\log \delta_{k}}{T B_{k}} \\
A T_{k}(i+1)=\left(1-\frac{1}{t_{c}}\right) A T_{k}(i)+I_{k}(i+1) * \frac{1}{t_{c}} * c q_{k}(i+1)
\end{gathered}
$$

where $\mu_{k}(i)$ is the priority of user $k$ at time $i, a_{k}$ is the desired QoS of user $k, \delta_{k}$ is the contentdependent packet loss threshold of user $k$, and $T B_{k}$ is the content-dependent buffer delay threshold of user $k$. It should be noted that the maximum residence time of RT/NRT content in the buffer at the base station is determined by content-dependent buffer delay threshold. $R T_{k}(i)$ is the residence time of the Head-of-Line (HOL) packet at the base station of user $k$ at time $i, c q_{k}(i)$ is the channel quality of user $k$ at time $i, A T_{k}(i)$ is the average throughput of user $k$ at time $i, t_{c}$ is a time constant and $I_{k}(i+1)$ is an indicator of the event where packets of user $k$ were transmitted at time $k+1$.

It can be observed in Eq. (1) that the packet scheduler in these single-user legacy wireless systems chooses only one user to receive its packets at each time. Moreover, in this system, all of the available bandwidth in a CC is used to transmit the packets to this user. This is contrary to packet scheduling in multi-user wireless systems that allows more than one user to receive its packets at each time.

Similarly in the multi-user wireless systems, a number of extensions of the well-known M-LWDF versions are available. This includes the algorithms developed in [3-5] that exploited the Resource Block (RB), the average throughput and the packet residence time to further improve the multi-user wireless performance. It should be noted that an RB is the smallest unit of radio resource in LTE-A. It contains twelve subcarriers of $15 \mathrm{kHz}$ bandwidth 
each and is of one ms interval. For example, the Modified Maximum-Largest Weighted Delay First (MM-LWDF) algorithm discussed in [3] uses Eq. (4) when determining the priority of each user on each $\mathrm{RB}$, on each $\mathrm{CC}$ and at each time.

$$
\mu_{k, l, m}(i)=a_{k} * R T_{k}(i) * \frac{c q_{k, l, m}(i)}{\sum_{l=1}^{l=C C_{\max }} A T_{k, l}(i)}
$$

where $\mu_{k, l, m}(i)$ is the priority of user $k$ on $\mathrm{CC} l$ on vacant $\mathrm{RB} m$ at time $i, a_{k}$ is the desired QoS of user $k$ as defined in Eq. (2), $R T_{k}(i)$ is the residence time of the HOL packet at the base station of user $k$ at time $i, c q_{k, l, m}(i)$ is the channel quality of user $k$ on $\mathrm{CC} l$ on vacant $\mathrm{RB} m$ at time $i, A T_{k, l}(i)$ is the average throughput of user $k$ on $C C l$ at time $i$ (as defined in Eq. (3)) and $C C_{\max }$ is the total number of licensed CCs.

Besides the extended versions of the M-LWDF, other studies that investigated the packet scheduling algorithms in the literature include [6-14]. Based on our study, it was observed that the majority of these conventional multi-user packet scheduling algorithms offer to meet the desired QoS of either for RT packets or NRT packets. Those PS algorithms that were developed for simultaneous support of both RT and NRT were computationally expensive, did not consider a realistic RT and NRT traffic, or require further performance investigation if these algorithms were to be implemented in the downlink cognitive LTE-A.

Due to the limitations of the conventional packet scheduling algorithms and motivated by the work in [3], this paper proposed an enhancement of the MM-LWDF known as an Enhanced M-LWDF (EM-LWDF) with the intention to provide support for more RT and NRT users that meet their desired QoS. The EM-LWDF algorithm takes the desired QoS of each packet (either RT or NRT), the channel quality, the average throughput and the urgency of each packet into consideration when scheduling packets on each RB and on each $\mathrm{CC}$.

The remaining parts of this paper are structured as follows: Section II explains in detail the proposed EM-LWDF algorithm, thereafter followed by the description of the simulation environment in Section III. Results are analytically discussed in Section IV whereas Section $\mathrm{V}$ marks the conclusions of the paper.

\section{ENHANCED MAXIMUM-LARGEST WEIGHTED DELAY FIRST (EM-LWDF) ALGORITHM}

The co-existence of the downlink LTE-A and DVB system as shown in Fig. 1 is considered in this paper. It should be noted that the base station of the LTE-A is known as eNodeB. It is assumed that the DVB system has a total of two licensed CCs of $3 \mathrm{MHz}$ bandwidth each. The CCs of the LTE-A are not taken into account as this paper aims to investigate the impact on cognitive LTE-A performance for opportunistic use of the vacant portions of the licensed DVB CCs via CR.

The EM-LWDF algorithm aims to simultaneously provide satisfactory QoS for both RT and NRT multimedia contents for more users. Therefore, to achieve its intention, the proposed algorithm selects the packets that maximize Eq. (5) at each time, on each licensed $\mathrm{CC}$ and on each vacant $\mathrm{RB}$. 


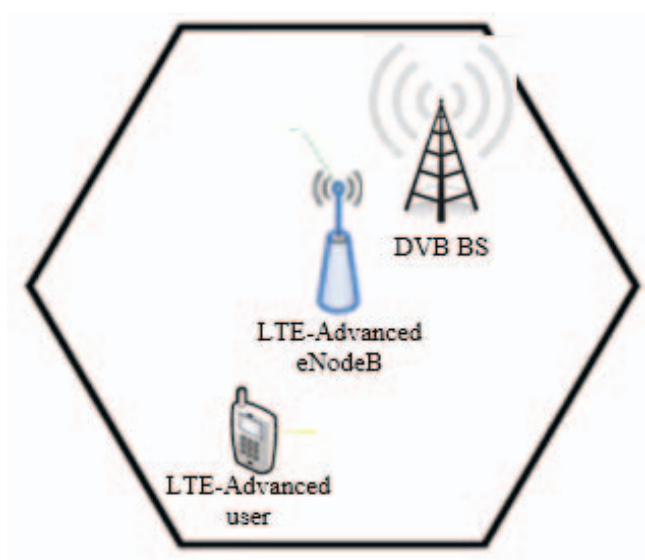

Fig. 1: Downlink cognitive LTE-A topology [15].

$$
\mu_{k, l, m}(i)=a_{k} * \frac{T B_{k i}(i)}{T B_{k}-R T_{k i}(i)} * \frac{c q_{k, l, m}(i)}{\sum_{l=1}^{l=C C_{\max }} A T_{k, l}(i)}
$$

where $\mu_{k, l, m}(i)$ is the priority of user $k$ on $\mathrm{CC} l$ on vacant $\mathrm{RB} m$ at time $i, a_{k}$ is the desired QoS of user $k$ as defined in Eq. (2), and $T B_{k}$ is the content-dependent buffer delay threshold of user $k$. $R T_{k}(i)$ is the residence time of the HOL packet at the eNodeB of user $k$ at time $i, c q_{k, l, m}(i)$ is the channel quality of user $k$ on $\mathrm{CC} l$ on vacant $\mathrm{RB} m$ at time $i, A T_{k, l}$ (i) is the average throughput of user $k$ on $C C l$ at time $i$ (as defined in Eq. (3)) and $C C_{\max }$ is the total number of licensed CCs.

The EM-LWDF has the following similarities with the MM-LWDF. It takes into account the desired QoS of each user, channel quality on each licensed $\mathrm{CC}$ and on each vacant RB as well as the average throughput on each licensed CC when making scheduling decisions. The desired QoS allows the EM-LWDF as well as the MM-LWDF to give a slightly higher priority for delay-sensitive RT packets whereas the channel quality enables the multiuser diversity to be exploited (i.e. a user with a good channel quality is favoured for packet transmission over the ones located at the cell edge. This allows the user to receive more packets as the channel quality on vacant RB used for packet transmission is good). If only the channel quality is accounted, then it will starve other users of poor channel qualities. Therefore, the average throughput on each licensed CC is accounted so as to increase fairness among the users by increasing the chances to users whose packets were not selected in the previous time to be scheduled in the consecutive time.

The main difference between the EM-LWDF and the MM-LWDF is the exploitation of the residence time of the HOL. In this case, the MM-LWDF only considers the packets that have resided the longest at the eNodeB $\left(R T_{k}(i)\right)$ whereas the EM-LWDF considers the outcome of $\frac{T B_{k}}{T B_{k}-R T_{k}(i)}$ so as to ensure that the most urgent packets are given a higher priority than the packets that have resided the longest at the eNodeB. This is one of the attempts made so as to reduce the number of discarded packets for violation of the content-dependent buffer delay threshold and hence reduce the RT and NRT packet loss 


\section{SIMULATION ENVIRONMENT}

The performance of the proposed EM-LWDF algorithm was evaluated on the basis of a Packet Loss Ratio (PLR) metric given that it is the most important metric when evaluating the QoS of multimedia performance. Definition of the PLR metric is given as follows:

$$
P L R=\frac{\sum_{k=1}^{K} \sum_{i=1}^{T} \operatorname{discard}_{-} \operatorname{packet}_{k}(i)}{\sum_{k=1}^{K} \sum_{i=1}^{T} \operatorname{arrive}_{-} \operatorname{packet}_{k}(i)}
$$

where $\operatorname{discard}_{\operatorname{packet}}(i)$ is the size of discarded packets of user $k$ at time $i$, $\operatorname{arrive~}_{\operatorname{packet}}(i)$ is the size of packets that arrived at the eNodeB buffer of user $k$ at time $i, K$ is the total number of users, and $T$ is the total simulation time.

The performance evaluation was conducted via a computer simulation and the simulation was run for a sufficient length of time to get stable results. The downlink cognitive LTE-A was modelled to contain only one eNodeB. This eNodeB is located at the centre of a cell of $450 \mathrm{~m}$ radius. Users are assumed to have uniform distribution within the cell and move at 30 $\mathrm{km} / \mathrm{h}$ speed. A total of two DVB CCs of $3 \mathrm{MHz}$ bandwidth is assumed and these CCs are of $700 \mathrm{MHz}$ and $704 \mathrm{MHz}$ bandwidths. Given that this paper aims to investigate the performance improvement due to RT and NRT multimedia scheduling in the cognitive LTEA system, the CCs of the LTE-A are not taken into account in this performance evaluation.

The content-dependent buffer delay threshold for RT and NRT contents are capped at 50 $\mathrm{ms}$ and $200 \mathrm{~ms}$ respectively. RT and NRT packets are discarded and considered as loss packets if their residence times exceed these thresholds. The PLR threshold for RT multimedia content is limited to $10^{-3}$ whereas $10^{-6}$ is the limiting PLR threshold for the NRT content. These thresholds are considered acceptable 3GPP thresholds. Moreover, it is assumed that the RT users are streaming video with $256 \mathrm{kbps}$ average data speed whereas the NRT users are browsing the web. It was assumed that the system has a variable number of RT and NRT users (i.e. 50:50, 75:25 and 25:75) to allow for the collection of more accurate simulation results.

\section{RESULTS AND DISCUSSION}

To assess the EM-LWDF, its performance was evaluated and compared with the wellknown Single-User M-LWDF [2] (also referred to as (Single Carrier M-LWDF, SCMLWDF) and MM-LWDF [3] algorithms. The SC M-LWDF was selected as the benchmark given its popularity and ability to meet the desired multimedia QoS in the single-user wireless systems. Moreover, to investigate M-LWDF in the multi-carrier wireless system, MM-LWDF was considered for benchmarking the EM-LWDF. The results are divided into three subsections for different distribution of RT and NRT cognitive LTE-A users.

\subsection{0:50 RT and NRT Cognitive LTE-A Users}

RT PLR of the EM-LWDF, SC M-LWDF and MM-LWDF algorithms with increasing number of cognitive LTE-A users is illustrated in Fig. 2. As previously discussed in Section 3 , the desired RT QoS is met if the PLR is kept below $10^{-3}$ (see Threshold line in the figure). The figure depicts that the EM-LWDF outperforms the SC M-LWDF and MM-LWDF in maximizing the number of cognitive LTE-A users at the required RT PLR. Table 1 shows that the EM-LWDF supports $185 \%$ and $9.6 \%$ more cognitive LTE-A users over the SC M- 
LWDF and MM-LWDF algorithms respectively. This improvement can be considered significant as the RT users are streaming video at $256 \mathrm{kbps}$ average data speed.

The NRT PLR performance with increasing number of cognitive LTE-A users of the EM-LWDF, SC M-LWDF and MM-LWDF are demonstrated in Fig. 3. When compared with the RT, the NRT content is more sensitive to lost packets. It requires its PLR to be maintained below $10^{-6}$ threshold. The figure demonstrates that with increasing number of cognitive LTE-A users, the EM-LWDF has a comparable performance with the MM-LWDF whereas the SC M-LWDF is the worst. However, the threshold of $10^{-6}$ threshold is exceeded by all three PS algorithms even for a small number of cognitive LTE-A users. This can be considered as acceptable because of the vacancy of the RBs in the downlink cognitive LTE-A (vacant RBs can only be used by the cognitive LTE-A users when licensed DVB users are not active and these cognitive LTE-A users have to compete for these scarce vacant RBs among themselves).

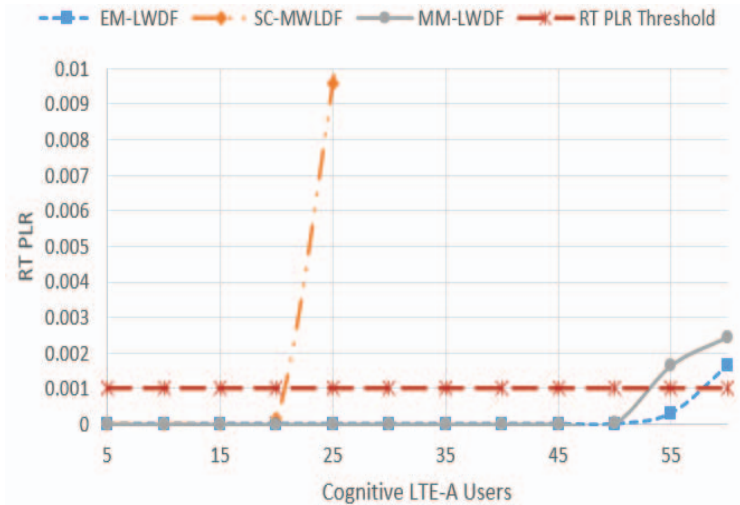

Fig. 2: RT PLR vs Cognitive LTE-A Users 50:50.

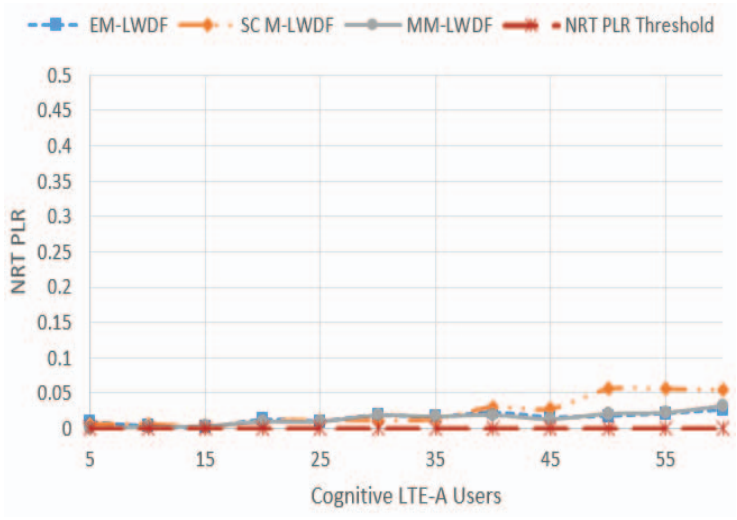

Fig. 3: NRT PLR vs Cognitive LTE-A Users 50:50.

Table 1: Maximum number of cognitive LTE-A users with satisfied QoS of RT contents $(50: 50)$

\begin{tabular}{ccc}
\hline PS Algorithms & $\begin{array}{c}\text { Maximum number of cognitive } \\
\text { LTE-A users }\end{array}$ & $\begin{array}{c}\text { Percentage of improvement } \\
\text { over benchmark PS } \\
\text { algorithms }\end{array}$ \\
\hline EM-LWDF & 57 & - \\
SC M-LWDF & 20 & $185 \%$ \\
MM-LWDF & 52 & $9.6 \%$ \\
\hline
\end{tabular}

\subsection{5:25 RT and NRT Cognitive LTE-A Users}

In Fig. 4, the RT PLR performance of EM-LWDF, SC M-LWDF and MM-LWDF are illustrated where $75 \%$ of the system capacity is RT cognitive LTE-A users and the remaining $25 \%$ is the NRT cognitive LTE-A users. The figure implies that EM-LWDF and MM-LWDF have a significantly greater number of RT cognitive LTE-A users that met the required RT PLR compared to SC M-LWDF. Based on Table 2, the EM-LWDF supports $200 \%$ and $15.4 \%$ more RT cognitive LTE-A users compared to SC M-LWDF and MM-LWDF, respectively. In comparison with the data in Table 1, the EM-LWDF improves 15\% and 5.8\% more RT users compared to SC M-LWDF and MM-LWDF respectively in maximizing RT cognitive LTE-A users at the required RT PLR. 


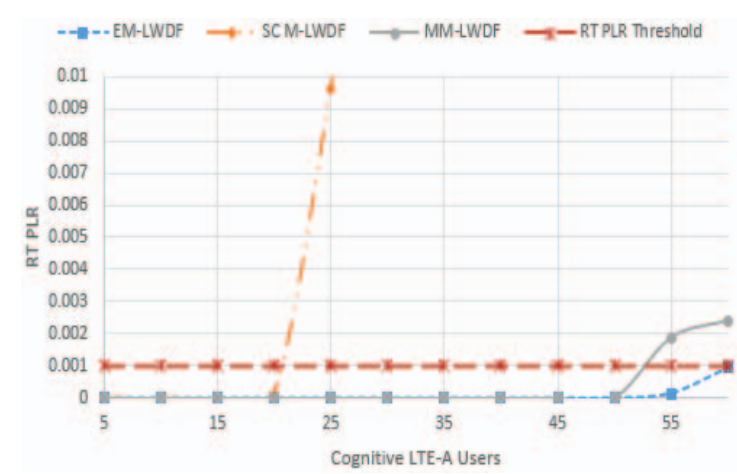

Fig. 4: RT PLR vs Cognitive LTE-A Users 75:25.

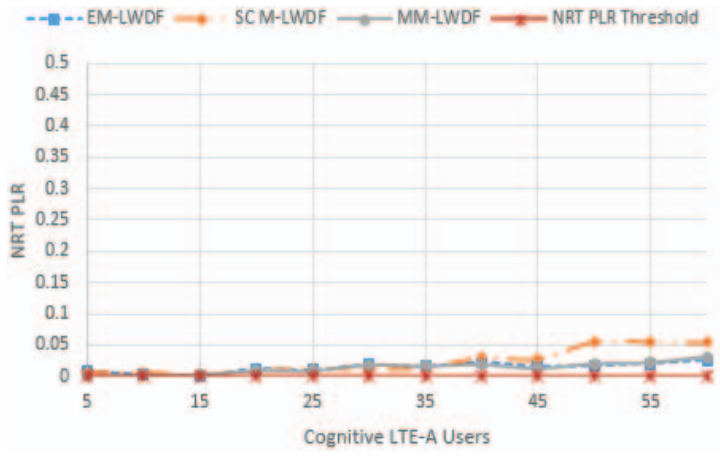

Fig. 5: NRT PLR vs Cognitive LTE-A Users 75:25.

Table 2: Maximum number of cognitive LTE-A users with satisfied QoS of RT contents $(75: 25)$

\begin{tabular}{ccc}
\hline PS Algorithms & $\begin{array}{c}\text { Maximum number of cognitive } \\
\text { LTE-A users }\end{array}$ & $\begin{array}{c}\text { Percentage of improvement } \\
\text { over benchmark PS } \\
\text { algorithms }\end{array}$ \\
\hline EM-LWDF & $>60$ & - \\
SC M-LWDF & 20 & $200 \%$ \\
MM-LWDF & 52 & $15.4 \%$ \\
\hline
\end{tabular}

The NRT PLR performance of EM-LWDF, SC M-LWDF and MM-LWDF when the distribution of RT and NRT cognitive LTE-A users is 75:25 is shown in Fig. 5. It is demonstrated in the figure that similar to the results achieved in sub-section 4.1, all algorithms exceed the NRT PLR threshold. This is due to the NRT contents being more sensitive to packet loss as compared to the RT. However, the figure shows that the EMLWDF has comparable performance as MM-LWDF while the SC M-LWDF significantly lags behind both algorithms.

\subsection{5:75 RT and NRT Cognitive LTE-A Users}

The RT PLR performance of EM-LWDF, SC M-LWDF and MM-LWDF with 25:75 distributions of RT and NRT cognitive LTE-A users are demonstrated in Fig. 6. It can be seen in the figure that EM-LWDF achieves similar performance compared to MM-LWDF and significantly outperforms the SC M-LWDF algorithm. Table 3 shows that EM-LWDF supports the same number of RT users as MM-LWDF and $130.8 \%$ more RT users compared to SC M-LWDF. The SC-MLWDF supports more users (i.e. 26 in 25:75 RT and NRT cognitive LTE-A users distribution as compared to 20 in 75:25 RT and NRT cognitive LTEA users distribution) because there are smaller number of RT users as compared to NRT users competing for the scarce vacant RBs.

Finally, the NRT PLR that indicates similar performances as in Fig. 3 and Fig. 5 are provided in Fig. 7. The NRT PLR threshold of $10^{-6}$ is more sensitive compared to the RT PLR threshold of $10^{-3}$ thus resulting in all of the three algorithms exceeding the NRT PLR threshold. However, EM-LWDF still manages to achieve comparable performance compared to MM-LWDF whereas the performance of the SC M-LWDF significantly degraded. Though the results indicate a fluctuation trend, this does not affect the overall performance of each algorithm given that the scale is very small. 


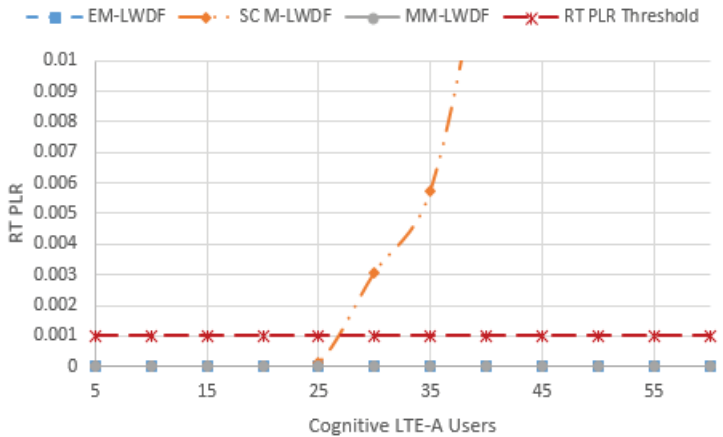

Fig. 6: RT PLR vs Cognitive LTE-A Users 25:75.

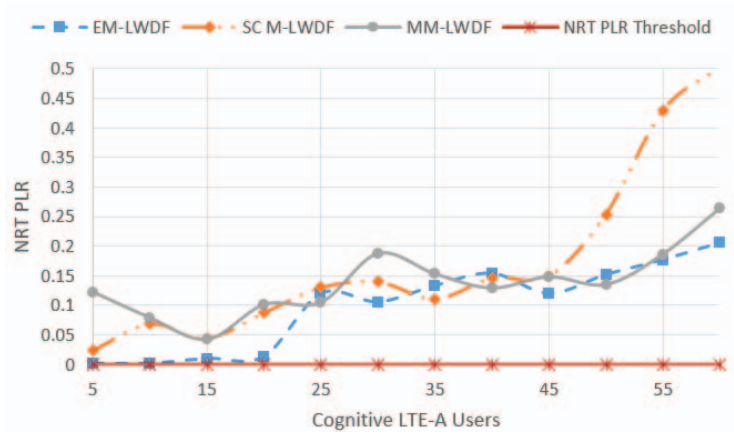

Fig. 7: NRT PLR vs Cognitive LTE-A Users 25:75.

Table 3: Maximum number of cognitive LTE-A users with satisfied QoS of RT contents $(25: 75)$

\begin{tabular}{ccc}
\hline PS Algorithms & $\begin{array}{c}\text { Maximum number of cognitive } \\
\text { LTE-A users }\end{array}$ & $\begin{array}{c}\text { Percentage of improvement } \\
\text { over benchmark PS } \\
\text { algorithms }\end{array}$ \\
\hline EM-LWDF & $>60$ & - \\
SC M-LWDF & 26 & $131 \%$ \\
MM-LWDF & $>60$ & $0 \%$ \\
\hline
\end{tabular}

The substantial improvement (especially in maximizing the number of cognitive LTE-A users with the desired RT QoS) of the EM-LWDF over the SC M-LWDF is because the SC M-LWDF assigns all of the vacant RBs to a selected user at each time and on each licensed CC. At a time, a number of vacant RBs in a licensed CC may not be utilized if the packets of the chosen user in the eNodeB buffer have all been transmitted. On the contrary, the EMLWDF allows efficient use of the vacant RBs at each time and on each licensed CC, by selecting a cognitive LTE-A user for packet transmission on each vacant RB.

The RT PLR of EM-LWDF is slightly better than the MM-LWDF because the algorithm always prioritizes cognitive LTE-A users with the most urgent packets (i.e. the residence time of the packets are approaching the content dependent-buffer delay threshold). This is one of the attempts made so as to reduce the RT PLR where it avoids RT packets from being discarded due to delay violation in the consecutive scheduling time. Even though there is a slight improvement achieved by the EM-LWDF as compared to the MM-LWDF, the EMLWDF does not introduce any new complexity in its algorithm given that the information of $T B_{k}$ and $R T_{k}$ are available at the eNodeB packet scheduler. Based on the results obtained in this performance evaluation it can be concluded that when compared with benchmark PS algorithms, the implementation of EM-LWDF in the downlink cognitive LTE-A system is more suited.

\section{CONCLUSION}

An enhanced packet scheduling algorithm known as EM-LWDF is proposed in this paper. The EM-LWDF algorithm was developed to prioritize the user with most urgent packet instead of the user with the packet that has waited the longest. In order to validate this improvement, several performance comparisons have been conducted. The results demonstrated that the proposed algorithm outperforms SC M-LWDF in supporting more 
cognitive LTE-A users at the required RT PLR where it supports $185 \%, 200 \%$, and $131 \%$ more cognitive LTE-A users in 50:50, 75:25, and 25:75 distributions of RT and NRT cognitive LTE-A users. The EM-LWDF algorithm also slightly outperforms MM-LWDF algorithm where it supports $9.6 \%$ and $15.4 \%$ more cognitive LTE-A users at required RT PLR in 50:50 and 75:25 distributions of RT and NRT cognitive LTE-A users and provides similar performance in the 25:75 situation. EM-LWDF achieves this feat without introducing any new complexity in its algorithm as compared to the MM-LWDF. Future works include enhancement of the EM-LWDF such that the desired QoS of the RT and NRT multimedia content can be simultaneously met. Additionally, the performance of the EM-LWDF will be compared with other packet scheduling algorithms that were developed for downlink cognitive LTE-A that simultaneously support RT and NRT multimedia contents. Performance evaluation of the EM-LWDF in multiple hexagonal cells taking the mobility and handover into account as well as with larger number of users (not capped at 60) will also be a part of future study.

\section{ACKNOWLEDGEMENT}

This research is funded by the Kementerian Pendidikan Malaysia under the Grant ID of FRGS/1/2019/TK04/UIAM/02/2.

\section{REFERENCES}

[1] Osa V, Herranz C, Monserrat JF, Gelabert X. (2012) Implementing Opportunistic Spectrum Access in LTE-Advanced. EURASIP Journal on Wireless Communications and Networking, 2012(1): 1-17.

[2] Andrews M, Kumaran K, Ramanan K, Stolyar A, Whiting P, Vijayakumar R. (2001) Providing Quality of Service over a Shared Wireless Link. IEEE Communications Magazine, 39(2): 150154.

[3] Ramli HAM. (2014) Performance of Maximum-Largest Weighted Delay First Algorithm in Long Term Evolution-Advanced with Carrier Aggregation. 2014 IEEE Wireless Communications and Networking Conference (WCNC): 1415-1420.

[4] Aiyetoro G, Takawira F. (2015) A New User Scheduling Scheme in LTE/LTE-A Networks using Cross-Layer Design Approach. MILCOM 2015 - 2015 IEEE Military Communications Conference: $1-5$.

[5] Aiyetoro G, Takawira F. (2014) A Cross-Layer Based Packet Scheduling Scheme for Multimedia Traffic in Satellite LTE Networks. 2014 6th International Conference on New Technologies, Mobility and Security (NTMS): 1-6.

[6] Bahreyni MS, Sattari-Naeini V. (2014) Fairness Aware Downlink Scheduling Algorithm for LTE Networks. Journal of Mathematics and Computer Science, 11(2): 53-63.

[7] Alfayly A, Mkwawa IH, Sun L, Ifeachor E. (2015) QoE-driven LTE downlink scheduling for VoIP application. 2015 12th Annual IEEE Consumer Communications and Networking Conference (CCNC): 603-604.

[8] Chung YL, Jang LJ, Tsai Z. (2011) An Efficient Downlink Packet Scheduling Algorithm in LTE-Advanced Systems with Carrier Aggregation. 2011 IEEE Consumer Communications and Networking Conference (CCNC): 632-636.

[9] Mnif K, Masmoudi A, Kamoun L. (2014) Adaptive Efficient Downlink Packet Scheduling Algorithm in LTE-Advanced System. The 2014 International Symposium on Networks, Computers and Communications: 1-5.

[10] Li J, Xu B, Xu Z, Li S, Liu Y. (2006) Adaptive Packet Scheduling Algorithm for Cognitive Radio System. International Conference on Communication Technology: 1-5.

[11] Mansor MIH, Ramli HAM, Asnawi AL, Isa FNM, (2017) Simulation of Packet Scheduling in Cognitive Long Term Evolution-Advanced. Indonesian Journal of Electrical Engineering \& Computer Science 8(2): 533-540. 
[12] Zhang Y, Zhang Y, Teng Y, Song M (2014) An Efficient Carrier Scheduling Scheme in Cognitive LTE-Advanced System with Carrier Aggregation, IEEE 25th Annual International Symposium on Personal, Indoor, and Mobile Radio Communication (PIMRC): 1970-1974.

[13] Vijayarani R, Nithyanandan L, (2017) Dynamic Slot-Based Carrier Scheduling Scheme for Downlink Multimedia Traffic Over LTE Advanced Networks with Carrier Aggregation. Turkish Journal of Electrical Engineering \& Computer Sciences, 25(4): 27962808.

[14] Kaur R, Kaur N, (2016) A Delay Reduced Scheduling Approach for Fast Spectrum Access in Cognitive Radio. International Journal of Engineering Development and Research, 4(2) 19461955.

[15] Ramli HAM, Isa FNM, Asnawi AL, Jusoh AZ, Azman AW, (2019) Urgency-Aware Scheduling Algorithm for Downlink Cognitive Long Term Evolution-Advanced. IEEE 89th Vehicular Technology Conference (VTC2019-Spring): 1-6. 